\title{
The Gravity Environment in Space Experiments
}

JackJ. W. A. van Loon

\section{1}

\section{Introduction to Gravity Research}

In 1687, Isaac Newton (1642-1727) produced his famous and important work Principia, based on the experiments and analyses of Galileo Galilei (1564-1642). In this work he proposed three main laws. The first was the law of inertia. He used the term inertia for the property of matter that causes it to resist a change in its state of motion. Inertia of an object must be overcome to set it in motion. The measure of an objects' inertia is its mass. The law of inertia states:

If the net force on an object is zero (i.e. if the vector sum of all forces acting on the object is zero), then the acceleration of the object is zero and the object moves with constant velocity.

$$
F_{\text {net }}=0
$$

Newton's second law states:

The accelerated motion of a body can only be produced by the application of a force to that body. The direction of the acceleration is the same as the direction of the force and the magnitude of the acceleration is proportional to the magnitude of the force.

$$
F=m a
$$

Commonly, $F$ is referred to as weight, $w$. The acceleration, $a$, is referred to as $g$ or gravity, on Earth $9.81 \mathrm{~m} \mathrm{~s}^{-2}, m=$ mass $(\mathrm{kg})$. The SI unit for gravity is "g" (lower case), not the often, especially in human gravitational physiology papers, applied " $G$ " that is in SI units the gravitational constant [see Eq. (4)].

Newton's third law tells that single forces cannot occur. Forces always act in pairs. It states: 
If object 1 exerts a force on object 2, then object 2 exerts an equal force, oppositely directed, on object 1 .

$$
F_{12}=-F_{21}
$$

All forces have a direction and a magnitude; force is a vector quantity. The SI unit of force $(\mathrm{F})$ is the newton. One newton $(\mathrm{N})$ is the unit mass $(\mathrm{kg})$ times the unit acceleration $\left(\mathrm{m} \mathrm{s}^{-2}\right)$. Obviously, these three laws also apply to microgravity or, better, "microweight" research, or in more general terms to acceleration research.

Gravity is one of the four basic forces described in nature today, besides the weak nuclear force, the strong nuclear force and the electrostatic force. Among these forces, gravity is by far the weakest. Compared with a unit gravity or hypergravity environment, various physical phenomena behave differently in the, theoretical, state of zero acceleration, also known as weightlessness. In our universe, zero gravity exists only in theoretical terms, since there is mass in outer space and, hence, gravity fields, as shown by Newton's "universal law of gravitation”:

$$
F_{\mathrm{G}}=G\left(m M / r^{2}\right)
$$

where $F_{\mathrm{G}}=$ the gravitational force, $G=$ the gravitational constant $\left(6.67 \times 10^{-11} \mathrm{~N} \mathrm{~m}^{2}\right.$ $\left.\mathrm{kg}^{-2}\right), m=$ mass of the object, $M=$ mass of the Earth, $r=$ distance between the centre of the two masses $m$ and $M$.

As mentioned before, in our Universe, there is no such thing as zero gravity, since masses are present everywhere and, therefore, so are gravitational forces. In an orbiting spacecraft such as the Space Shuttle, Soyuz or the International Space Station there is also a small gravity residuum due to various reasons. A typical spacecraft would experience atmospheric drag $\left(6-30 \cdot \times 10^{-7} \times \mathrm{g}\right.$, at $250 \mathrm{~km}$ altitude), solar radiation pressure $\left( \pm 5 \cdot \times 10^{-9} \times \mathrm{g}\right)$, gravity gradient of extended bodies not located in the centre of mass $\left( \pm 5 \cdot \times 10^{-9} \times \mathrm{g}\right)$ and finally self-gravitation due to its own mass $\left( \pm 10^{-9} \times g\right)$ [1]. Disturbance of the gravity level in space flight experiments is further discussed below.

\subsection{1}

\section{Principle of Equivalence}

An important aspect of Einstein's general relativity has to do with the equivalence of gravitational fields and accelerated motion. If we are in a laboratory on Earth, a mass that is released will fall, or accelerate, downward due to the gravitational attraction of the Earth. Now, let us, theoretically, move this laboratory into space, away from the gravitational influence. We now take the same object and release it within an accelerating rocket. Presume the level of acceleration of the rocket is the same as the Earth gravity, $g\left(9.81 \mathrm{~m} \mathrm{~s}^{-2}\right)$. In such a situation the rocket will "push" onto the laboratory floor and move this floor towards the "falling" mass. As far as the observations of the two motions of the mass relative to the floor are concerned, the accelerated motion in the two cases will be exactly the same. If the 


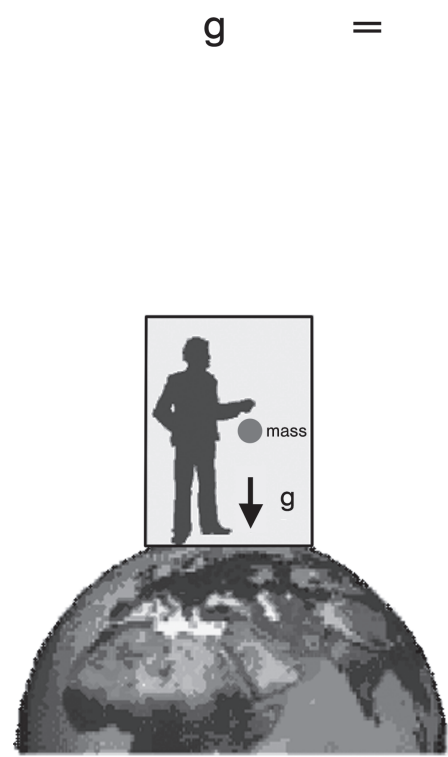

Gravitational mass

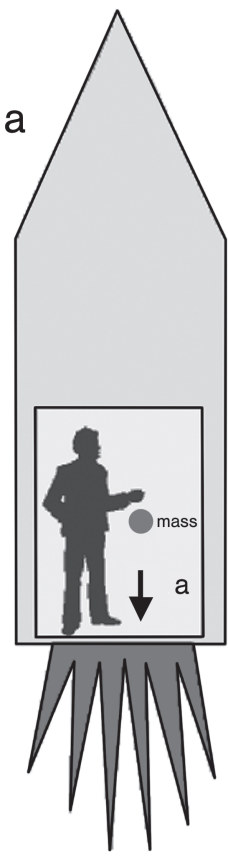

Inertial mass

Fig. 1.1 Difference between "gravitational mass" $\left(m_{\mathrm{C}}\right)$ and "inertial mass" $\left(m_{1}\right)$. The observer would see no difference between the two accelerations, hence $m_{G}=m_{1}$. (Picture used with permission from J. van Loon, DESC, Amsterdam.)

laboratory had no windows, the observer could not distinguish between an acceleration due to gravity (e.g. on Earth) and an acceleration due to a "push" of, for example, a rocket in space (Fig. 1.1). Or, as Einstein described exactly one century ago in his "principle of equivalence":

In a closed laboratory, no experiment can be performed that will distinguish between the effects of a gravitational field and the effects due to an acceleration with respect to some inertial reference frame.

\section{1 .2}

\section{Microgravity}

One of the often made mistakes about microgravity and space flight is to presume that the presence of microgravity in an orbiting spacecraft, such as the Space Shuttle or the International Space Station, ISS, is because "they are in Space" or "outside the Earth's atmosphere", although it might seem that these spacecraft are "far" away from human perspective they are actually quite close to Earth (only $300-500 \mathrm{~km}$ above the Earth's surface). So it is not the fact of being up in Space 
but rather that a spacecraft is orbiting at a particular velocity that provides a near weightlessness environment. These spaceships or satellites actually fall around the Earth with a speed of about $28000 \mathrm{~km} \mathrm{~h}^{-1}$. In fact, if the Earth's surface would be as smooth as a snooker ball it would be possible to provide microgravity in a capsule that circulates the Earth's surface at a distance of just $1 \mathrm{~mm}$. For a typical Shuttle mission at an altitude of, for example, $350 \mathrm{~km}$ the level of gravity is still $9.04 \mathrm{~m} \mathrm{~s}^{-2}$ that means only $8 \%$ less than the gravitational field on the Earth's surface.

It was only at the onset of space exploration and with the possibility of performing experiments in free falling capsules that we became able to perform so-called "microgravity" or better "microweight" experiments. The possibility to compensate the, on Earth, ever present gravitational force provides us with a very useful tool and a unique experiment environment to study the effects of gravity on fluids, dust or plasmas, but also possible effects on living systems such as humans, animals, plants, cells or even smaller structures.

Before we could actually perform spaceflight experiments, there were some reports that stated that, on a cellular level, gravity, either micro- or hypo- or hypergravity, would have no effect on these systems. One of the first papers on the physical background of microgravity studies on a cellular level was written by Pollard over 40 years ago [2]. In this very illustrative work he evaluates phenomena like temperature, Brownian movement, convection, hydrostatic forces, and stresses experienced by a cell membrane. He concluded that cells with a diameter of $10 \mu \mathrm{m}$ and more would experience gravity. This effect could be due do the redistribution of nucleoli or mitochondria. Although comparing various forces and energies present within living cells may be very illustrative, their magnitude is not necessarily related to their impact. The extent of various cellular physicochemical phenomena has been described in an interesting essay by Albrecht-Buehler [3]. In this paper the author relates the micro-environment of cells with the macroenvironment where we, as human beings, relate to. It is calculated that the force of gravity is $400000 \times$ smaller than the force of surface tension, and that the force for moving only one single electron in a typical electrical field of a nerve cell membrane is comparable to the weight of an entire cell. Water appears to be very viscous on a cellular level, while the impact energy, by Brownian movement, of only three water molecules is comparable to the weight of an entire cell. The gravitational potential energy of a cell due to gravity is about $21 \mathrm{~kJ} \mathrm{~mol}^{-1}$, while the chemical energy of only one hydrogen bond is $17 \mathrm{~kJ} \mathrm{~mol}^{-1}$. Finally, the contractile force of a single sarcomere is comparable to the weight of 60 cells. In addition, the force of polymerization is far more powerful than the weight of a single cell, while the force needed to add only one sub-unit of, for example, microfilaments, is about ten times larger than the weight of a cell.

All these theoretical studies indicate that, on a (sub-)cellular level, gravity would have no impact whatsoever. However, in recent decades numerous studies, both on orbiting spacecraft and in ground-based facilities, have indicated that gravity does have an effect on small systems (for reviews see Refs. [4-11]). The mechanism of this phenomenon is still a matter of debate. More recent experiments and, 
especially, future experiments on board of orbiting spacecrafts as well as in groundbased facilities might reveal some of the mechanisms involved.

\section{1 .3}

\section{Artificial Gravity}

Since gravity, or acceleration, is a vectorial entity we can produce "artificial gravity" by changing the direction of the vector. This can be done in a centrifuge. In a constantly rotating centrifuge the object moves with a constant velocity. However, since the orientation is constantly changed, the object is accelerated:

$$
a_{\mathrm{c}}=\omega^{2} r
$$

where $a_{\mathrm{c}}=$ centripetal acceleration $\left(\mathrm{m} \mathrm{s}^{-2}\right), \omega=\operatorname{angular}$ velocity $\left(\operatorname{rad~s}^{-1}\right), r=$ radius (m).

The force on this object would be a centripetal force:

$$
F_{\mathrm{c}}=m a_{\mathrm{c}}=m \omega^{2} r
$$

In spaceflight experiments, especially in biology, we often make use of a socalled "on-board" centrifuge. Why would we spend so much effort and money to do a spaceflight/near weightlessness experiment but also provide a $1 \times g$ environment?

To be able to draw any conclusions from spaceflight, which requires microgravity experiments, it is important to have a proper control group. To obtain the best control, we have to fully understand all possible influences involved in such experiments. What is that proper control? There are some important differences between a sample exposed to spaceflight microgravity $(\mu \mathrm{g})$ and a $1 \times \mathrm{g}$ on-ground control, such as launch vibrations, cosmic radiation and experiment lag time. The better control seems to be an on-board centrifuge. See also Table 1.1.

For biological experiments it was mainly the Biorack facility $[4,12]$ and later many others that accommodated an on-board $1 \times g$ control. However, even though an on-board centrifuge might be the best control, such a configuration also brings about particular artefacts between ground and flight $1 \times \mathrm{g}$, one of them being the inertial shear forces [13, 14].

Shear forces can be brought about by inertia (inertial shear) and/or fluid flow (fluid shear). In cell biology, fluid shear is an important physiological phenomenon and most common in blood vessels, where endothelial cells are exposed to blood flow. For endothelial cells the shear stress is in the order of 0.1-0.5 and 0.6-4.0 Pa in venous and arterial vessels, respectively [15]. Not only the cardiovascular system but also the mechano-adaptation of bone is most likely governed by fluid shear forces around osteocytes [16, 17 and Chapter 6]. Inertial shear forces, however, are mostly generated in materials exposed to accelerations. In cells, both fluid shear stress and inertial shear stress will generate cell deformation or strain. In centrifuges, an essential difference between inertial shear force, $F_{\mathrm{i}}$, and 
Table 1.1 Various parameters experienced by samples in-flight

( $1 \times \mathrm{g}$ controls and microgravity, $\mu \mathrm{g}$ ) as compared with on-ground samples.

\begin{tabular}{|c|c|c|c|}
\hline Variable & On-ground $1 \times g$ & In-flight $1 \times g$ & In-flight $\mu \mathrm{g}$ \\
\hline Launch accelerations & No & Yes & Yes \\
\hline Launch vibrations ${ }^{\mathrm{a}}$ & No & Yes & Yes \\
\hline Cosmic radiation & No & Yes & Yes \\
\hline Need for centrifuge & No & Yes & NA \\
\hline Centrifuge vibration ${ }^{\mathrm{b}}$ & No & Yes & No \\
\hline $\begin{array}{l}\text { Exposure to microgravity before start of } \\
\text { experiment }{ }^{c}\end{array}$ & No & Yes & Yes \\
\hline Temperature gradients/differences ${ }^{\mathrm{d}}$ & Different & Different & Different \\
\hline Storage conditions ${ }^{\mathrm{e}}$ & Different & Different & Different \\
\hline Flight Crew procedures and manipulations ${ }^{\mathrm{e}}$ & No & Yes & Yes \\
\hline Inertial shear forces & No & Yes & No \\
\hline
\end{tabular}

a Launch vibrations are also accelerations, being at higher frequencies and in random directions.

b Static, non-rotating, samples may still be exposed to remnant vibrations of a centrifuge or other moving parts transmitted through the structure of the facilities or the spacecraft.

c For some facilities, like Biobox, or Kubik, this time is reduced to a minimum.

d Small temperature gradients between, for example, a centrifuge and static sample, or between ground and flight samples.

e Depending on the environment and crew-members involved, there will always be (small) differences in experiment and sample handling.

the force of gravity, $F_{\mathrm{g}}$, is that inertial shear acts perpendicular to the gravity acceleration vector (Fig. 1.2).

The level of inertial shear experienced by an adherent cell layer depends on the radius of the centrifuge and the location of the cells within the sample surface area. Adherent cells attached to a flat surface will experience a larger inertial shear force, $F_{\mathrm{i}}$, when located further from the point where the radius is perpendicular to the monolayer surface (Fig. 1.2). This effect is enhanced in centrifuges with a smaller radius. Inertial shear acceleration for adherent cells results in cell deformation that means strain.

For non-adherent and non-actively moving cells the situation is different. Here the cells do not experience inertial shear strain, as they do not attach to the substrate. However, cells on an in-flight $1 \times$ g centrifuge arrange themselves differently in the sample volume than they would on Earth. Free moving particles in a rotating system will move to the area of highest acceleration. When we consider a homogeneous suspension of cells and place this, on-ground, in a flat bottomed dish the cells will distribute evenly over the surface area as shown in Fig. 1.3(A). When we apply $1 \times g$ to the same dish in an on-board centrifuge the cells will, due to inertial force, move to the experiment container outer edges. In such a configuration the cells will pile up onto each other (Fig. 1.3B). One could imagine that all kind of cells physiological properties might change because of this. Cells might have more cell-cell interactions and medium depletion might become an issue, 


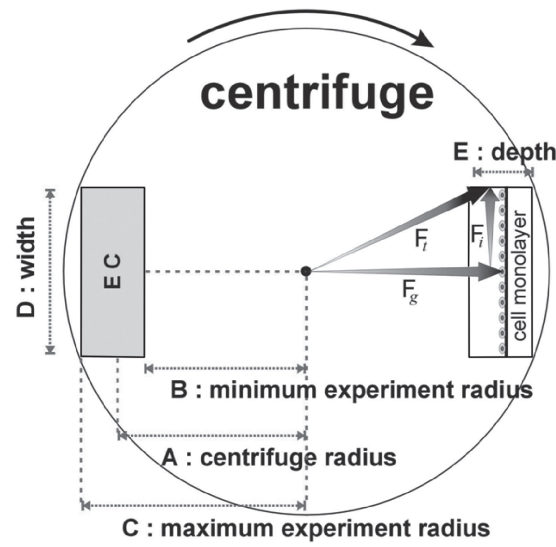

Fig. 1.2 Geometry of an Experiment Container (EC) accommodated on a centrifuge and forces within such a rotating system on board a spacecraft in free fall (weightlessness) condition. The centrifuge radius, $A$, is defined as the distance from the centre of rotation to the centre of the EC. The minimum radius, $B$, is the distance from the centre of rotation to the inner wall of the EC. The maximum radius, $C$, is the distance from the centre of rotation to the outer wall of the EC. Width, $D$, is the maximum lateral width of an EC. The force of gravity, $F_{\mathrm{g}}$, increases radially from the centre of centrifugation. The inertial shear force, $F_{\mathrm{i}}$, increases laterally from the centre of the EC, as depicted in the right-hand EC along a plane surface with a schematic monolayer of cells. (Picture adopted from Ref. [13] with kind permission from the Journal of Biomechanical Engineering, American Society of Mechanical Engineers, ASME, New York.)

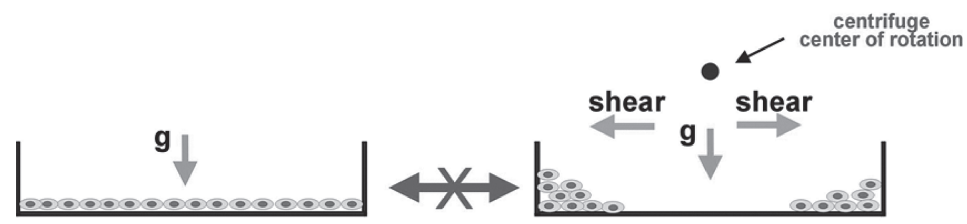

A: on-ground static $(1 \times g)$

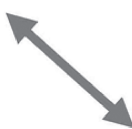

$B$ : in-flight centrifuge $1 \times g$

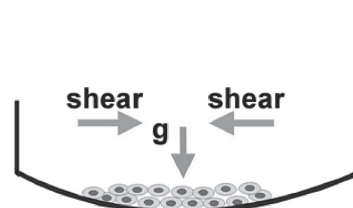

C: on-ground static $(1 \times g)$

Fig. 1.3 Distribution of non-adherent cells in a $1 \times g$ static on-ground environment $(A$ and $C)$
or on a $1 \times g$ on-board centrifuge $(B$ and $D)$ in sample chambers of different surface geometry. Note that the mark for the "centre of rotation" and the curvature of the chamber
D: in-flight centrifuge $\mathbf{1} \times \mathbf{g}$

are for clarity of the drawing and are not on the same scale. (Picture adopted from Ref. [13] with kind permission from the Journal of Biomechanical Engineering, American Society of Mechanical Engineers, ASME, New York.) 
especially in microgravity when there is no convection. Therefore, the design of flight hardware, but also modules used for ground based microgravity simulations such as in a Random Positioning Machine, should ideally reflect these considerations to provide optimal culture conditions to the samples.

1.2

\section{Gravity Phenomena on Small Objects}

Several phenomena are expressed differently or have another order of magnitude in an acceleration field (e.g. 1 $\times$ g Earth's gravity) as compared with a near weightlessness condition. When thinking about any effects of gravity on, e.g. a biological cell, one should distinguish between direct and indirect effects.

Direct effects may be described as effects of acceleration directly onto the cell or its internal components. Indirect effects are effects mediated to the cell by its environment. Some effects reported in cell culture experiments might be interpreted as "direct" while "indirect" effects might not be excluded.

When considering gravitational research we have to be aware of several phenomena that play a role, especially at the level of a biological cell with in vitro experiments (i.e. in a "Petri-dish").

\subsection{1}

\section{Sedimentation}

Sedimentation is the downward movement (along the gravity vector $\mathrm{g}$ ) of an object in such a way that the object moves relative to its surrounding medium. During this process the medium, such as a fluid, exerts a force on the object known as drag force. This drag force is due to the viscosity and also, at high speeds, to turbulence behind the object.

By definition, the sedimentation force $\left(F_{\mathrm{S}}\right)$ is the downward force of weight due to linear acceleration $(m g)$ minus buoyancy $\left(F_{\mathrm{B}}\right)$ minus frictional forces opposing downward motion $\left(F_{\mathrm{F}}\right)$ :

$$
\begin{aligned}
& F_{\mathrm{S}}=m g-F_{\mathrm{B}}-F_{\mathrm{F}} \\
& F_{\mathrm{S}}=m g-\left(\rho_{\mathrm{f}} V g\right)-(6 \pi r \eta v)
\end{aligned}
$$

where $m=$ mass of the object $(\mathrm{kg}), g=$ the gravity acceleration $\left(\mathrm{m} \mathrm{s}^{-2}\right), F_{\mathrm{B}}=$ buoyant force (see below) $=\rho_{\mathrm{f}} V \mathrm{~g}$, with $\rho_{\mathrm{f}}=$ specific density of the fluid $\left(\mathrm{g} \mathrm{cm}^{-3}\right), V=$ volume of the displaced fluid (=volume of the object) $\left(\mathrm{m}^{3}\right)$.

$$
F_{\mathrm{F}}=\text { frictional force, see Stokes equation }[\text { Eq. }(17)]=k v
$$

where $k$ from the Stokes equation [Eq. (17)] $=6 \pi r \eta$ (for a spherical object), with $r=$ radius of the object $(\mathrm{m}), \eta=$ viscosity constant $\left(\mathrm{Nsm}^{-2}\right.$ or Pas $), v=$ object's velocity relative to the fluid $\left(\mathrm{m} \mathrm{s}^{-1}\right)$. 
Sedimentation is one of the main differences between the experimental environment on Earth and in space. From daily experience we know that big $(>r)$ and heavy $(>\rho)$ particles are the quickest settlers.

These relatively big and heavy particles play a role in the positive gravitropism of plant roots. The so-called amyloplasts, localized in specialized cells in the root tip, the statocytes, sediment to the bottom of the cell in response to gravity (for review see Ref. [19]). Also, for the fresh water unicellular ciliate Loxodes it is argued that the heavy cell compartments (Mueller organelles), containing barium sulfate crystals, may be a good candidate for the gravisensor in these species (for review see Ref. [9]).

\subsection{2}

\section{Hydrostatic Pressure}

Hydrostatic pressure is also influenced by gravity. In situations of hypergravity part of the pressure that acts on a surface area derives from the weight of the liquid column standing above these surfaces, such as the culture medium above a monolayer of cells. Pressure is defined as force per unit area, where the force, $F$, is always acting perpendicular to that surface. The SI unit for pressure is the Pascal $(\mathrm{Pa})$, one Pascal being one Newton per square meter $\left(\mathrm{N} \mathrm{m}^{-2}\right)$.

In liquids or gasses of constant density:

$$
\begin{aligned}
P & =F / A \\
& =\rho A g h / A
\end{aligned}
$$

where $P=$ pressure $(\mathrm{Pa}), F=$ force $(\mathrm{N}), A=$ surface area $\left(\mathrm{m}^{2}\right), \rho=$ density of the liquid or gas $\left(\mathrm{kg} / \mathrm{m}^{3}\right), g=$ unit gravity $\left(\mathrm{m} \mathrm{s}^{-2}\right), h=$ height of the liquid (or gas) column (m).

Normally, the pressure exerted by a fluid column is additional to atmospheric pressure. The total pressure would be:

$$
P_{\text {tot }}=\rho g h+P_{\mathrm{o}}
$$

where $P_{\mathrm{o}}=$ atmospheric pressure $(\mathrm{Pa})$. Clearly, from this equation, hydrostatic pressure is linearly proportional to g. Under pure weightlessness conditions, hydrostatic pressure due to weight is zero. One should keep in mind, though, that in manned missions the spacecraft interior pressure exerts a hydrostatic pressure in a liquid. During hypergravity experiments, however, in which cells were cultured at $320 \times \mathrm{g}$ [20] with an assumed liquid column above the cells of $1 \mathrm{~mm}$, the hydrostatic pressure on the surface of the culture plate due to liquid weight would be almost $3.3 \mathrm{kPa}$ (see calculations below), and about $10 \mathrm{kPa}$ with a $3 \mathrm{~mm}$ liquid column. In addition to the pressure deriving from the liquid column, the atmospheric pressure should also be taken into account. Atmospheric pressure, also referred to as one atmosphere, is about $100 \mathrm{kPa}(=1000 \mathrm{hPa})$ at sea level. 


$$
\begin{aligned}
P & =\rho g h \\
& =1.05 \times 10^{3} \mathrm{~kg} \mathrm{~m}^{-3} \times 9.81 \mathrm{~m} \mathrm{~s}^{-2} \times 320 \times 0.001 \mathrm{~m} \\
& =3.296 \mathrm{kPa}
\end{aligned}
$$

where $\rho=$ specific density of culture medium $\cong 1.05 \mathrm{~kg} \mathrm{~m}^{-3}, \mathrm{~g}=320 \times$ unit gravity $\left(\mathrm{m} \mathrm{s}^{-2}\right), h=1 \mathrm{~mm}$ liquid column above the cell layer $(\mathrm{m})$.

In Inoue's experiment in the $320 \times g$ group [20], the force due to hydrostatic pressure acting upon a particular cell surface in a monolayer culture with a cell diameter of $10 \mu \mathrm{m}$ would be around $2.6 \times 10^{-7} \mathrm{~N}$. In comparison, the force of the same cell due to its own weight would be around $5.1 \times 10^{-12} \mathrm{~N}$. This indicates that hydrostatic pressures cannot be neglected when considering cells subjected to hypergravity conditions.

\section{2 .3}

\section{Diffusion}

According to kinetic theories, the molecules in a gas or in a liquid are continuously moving around and are colliding with each other. This is the process of diffusion. It can also be visualized microscopically, and is better known as the Brownian movement of small particles in solution. The diffusion process is similar to the conduction of heat through a mass. The conduction of heat takes place in response to a temperature gradient while diffusion takes place in response to a concentration gradient. The physiologist Adolf Fick formulated his equation for diffusion in 1855 :

$$
\Delta N / \Delta t=-D A \times(\Delta C / \Delta x)
$$

where $\Delta N=$ number of diffusing molecules (n), $\Delta t=$ time needed for diffusion (s), $A=$ surface area on which diffusion takes place $\left(\mathrm{m}^{2}\right), \Delta C=\left(C_{1}-C_{2}\right)$ concentration difference at two locations within the surface area, $\Delta x=$ distance between $C_{1}$ and $C_{2}(\mathrm{~m}), D=$ the diffusion coefficient for a particular substance:

$$
D=(k T / 4 \pi \eta r)
$$

where $k=$ Boltzmann constant $\left(1.380658 \mathrm{~J} \mathrm{~K}^{-1}\right), T=$ temperature $(\mathrm{K}), \eta=$ viscosity constant $\left(\mathrm{Ns} \mathrm{m}^{-2}=\mathrm{Pas}\right)$ [2], $r=$ radius of diffusing molecule (assumed spherical) (m) - for a spherical particle:

$$
r=\left(3 M / 4 \pi \rho N_{\mathrm{A}}\right)^{1 / 3}
$$

where $M=$ mass per mole, $\rho=$ density of the molecule $\left(\mathrm{kg} \mathrm{L}^{-1}\right), N_{\mathrm{A}}=$ Avogadro constant $\left(\mathrm{mol}^{-1}\right)$.

The negative sign in $-D A$ reminds us that the flow of molecules is opposite to the concentration gradient. Diffusion is not altered under microgravity. Therefore, 
in the absence of sedimentation, diffusion is one of the main remaining in vitro "mixing facilities" on the molecular level under weightlessness conditions.

\section{2 .4}

\section{Convection}

Convection is the movement of fluid or gas that is based on density variations. When a part of a liquid or gas is heated up it expands, causing its density to decrease. In the same liquid this heated part moves upwards since it is lighter. This phenomenon is only present under conditions of acceleration (that means gravity) and is absent in weightlessness. The impact of density differences within a biological cell due to temperature differences is negligible, based on the high heat conductivity and relatively small temperature differences in cells [2]. The absence of convection during in vitro experiments in space could mean that spent culture medium and metabolic waste products, but also autocrine growth factors, accumulate around the cells and tissues under microgravity. In contrast, fresh nutrients from the culture medium are depleted and only supplied to the cell by diffusion processes. This could of course modulate growth and differentiation. The impact would be a secondary effect of microgravity on cell metabolism.

\section{2 .5}

\section{Diffusion/Convection}

The effects of diffusion and convection differ considerably in magnitudes. The following gives an idea of diffusion velocity times for non-colliding particles. It is based on Einstein's mean-square or "random-walk", $x^{2}$, diffusion distance. The diffusion coefficient of $\mathrm{K}^{+}$ions in water is, $D=1 \times 10^{-5} \mathrm{~cm}^{2} \mathrm{~s}^{-1}$ [21]. For a onedimensional displacement over $5 \mathrm{~mm}$ by diffusion it would take $\mathrm{K}^{+}$ions nearly:

$$
\begin{aligned}
& t=x^{2} / D \\
& t=25 / 10^{-3}=25000 \mathrm{~s}=6.9 \mathrm{~h}
\end{aligned}
$$

where $x=$ displacement distance $(\mathrm{mm})$ and $D=$ diffusion coefficient $\left(\mathrm{mm}^{2} \mathrm{~s}^{-1}\right)$. Table 1.2 gives some values of $D$.

Convection currents that result from density differences generate a larger mixing capacity. Assume a spherical particle with a radius $r=0.1 \mathrm{~mm}$ in water. The density of water $\rho=1 \mathrm{~g} \mathrm{~cm}^{-3}$ and the viscosity $\eta=0.01$ Poise. Assume the specific gravity of the particle is $1 \%$ more than water, $\rho=1.01 \mathrm{~g} \mathrm{~cm}^{-3}$. The Stokes equation for friction, $F$, would be:

$$
\begin{aligned}
& F=6 \pi r \eta v=(4 / 3) \pi r^{3}\left(\rho_{\text {water }}-\rho\right) g \\
& v=(2 / 9) \eta\left(\rho_{\text {water }}-\rho\right) r^{2} \mathrm{~g} \\
& v=0.2 \mathrm{~mm} \mathrm{~s}^{-1}
\end{aligned}
$$


Table 1.2 Some examples of diffusion rates.

\begin{tabular}{lccc}
\hline Particle/molecule & Diameter & Measured in & Diffusion coefficient (D) $\left(\mathbf{c m}^{2} \mathbf{s}^{-1}\right)$ \\
\hline Spherical particle & $1 \mathrm{~mm}$ & Water $\left(25^{\circ} \mathrm{C}\right)$ & $5 \times 10^{-12 \mathrm{a}}$ \\
Whole cell & $-10 \mu \mathrm{m}$ & - & $<10^{-10 \mathrm{~b}}$ \\
Spherical particle & $1 \mu \mathrm{m}$ & Water $\left(25^{\circ} \mathrm{C}\right)$ & $5 \times 10^{-9 \mathrm{a}}$ \\
Albumine & - & - & $6 \times 10^{-7 b}$ \\
Raffinose & - & Water $\left(15^{\circ} \mathrm{C}\right)$ & $3.3 \times 10^{-6 a}$ \\
Spherical particle & $1 \mathrm{~nm}$ & Water $\left(25^{\circ} \mathrm{C}\right)$ & $5 \times 10^{-6 \mathrm{a}}$ \\
Sucrose & - & Water $\left(25^{\circ} \mathrm{C}\right)$ & $5.2 \times 10^{-6 \mathrm{a}}$ \\
1-Butanol & - & Water $\left(25^{\circ} \mathrm{C}\right)$ & $5.6 \times 10^{-6 \mathrm{a}}$ \\
Glucose & - & Water $\left(25^{\circ} \mathrm{C}\right)$ & $6.7 \times 10^{-6 \mathrm{a}}$ \\
Ethanol & - & Water $\left(25^{\circ} \mathrm{C}\right)$ & $12.4 \times 10^{-6 \mathrm{a}}$ \\
Methanol & - & Water $\left(15^{\circ} \mathrm{C}\right)$ & $12.8 \times 10^{-6 \mathrm{a}}$ \\
Methane & - & Water $\left(25^{\circ} \mathrm{C}\right)$ & $14.9 \times 10^{-6 \mathrm{a}}$ \\
\hline
\end{tabular}

a Handbook Chemistry \& Physics [26].

b Todd, 1989 [27].

where $r$ =radius of particle $(\mathrm{m}), \eta=$ viscosity constant $(\mathrm{Pas}), v=$ velocity $\left(\mathrm{m} \mathrm{s}^{-1}\right)$, $\rho=$ density of particle $\left(\mathrm{g} \mathrm{cm}^{-3}\right)$

Or, within $6.9 \mathrm{~h}$ this particle would have travelled nearly 5 metres. In this example the convection process is nearly $1000 \times$ faster than diffusion.

\subsection{6}

\section{Buoyancy}

The phenomenon of buoyancy-driven convection, or Rayleigh convection, is absent in a weightlessness environment. Density differences that cause this convection are the result of local changes in temperature or composition. This is one of the important differences between ground $(1 \times g)$ and flight $(\mu g)$ experiments. The net force acting on an object in relation to its buoyancy is:

$$
F_{\mathrm{B}, \mathrm{net}}=m g+F_{1}-F_{2}
$$

where $m g$ =downwards force due to the object's weight, $F_{1}=$ downwards force due to the fluid on top of the object:

$$
=\rho_{\mathrm{f}} g h_{1} A
$$

$F_{2}=$ upwards force due to buoyancy:

$$
=\rho_{\mathrm{f}} g h_{2} A
$$

where $m=$ mass of the object $(\mathrm{kg}), \rho_{\mathrm{f}}=$ density of the fluid $\left(\mathrm{g} \mathrm{cm}^{-3}\right), g=$ the (gravity) acceleration $\left(\mathrm{m} \mathrm{s}^{-2}\right), h_{1}=$ height of the fluid column above the object $(\mathrm{m}), h_{2}=$ height 
of the fluid column from the surface of the fluid to the bottom of the object $(\mathrm{m})$, $A=$ surface area (of a cylinder or cube) $\left(\mathrm{m}^{2}\right)$.

For a hypothetical cylindrical shaped object, $\left(h_{2}-h_{1}\right) A=V$ is the volume of this cylinder and $\rho_{\mathrm{f}} \times V=M$ is the mass of the fluid with a volume equal to the volume of the object:

$$
\rho_{\mathrm{f}} g\left(h_{2}-h_{1}\right) A=M g=w_{\mathrm{f}}
$$

where $w_{\mathrm{f}}$ is the weight of the fluid that has been displaced by the object. Thus:

$$
F_{\mathrm{B}, \text { net }}=m g-w_{\mathrm{f}}
$$

The force of buoyancy of an object works opposite to gravity and is proportional to the object's volume and the liquid's weight. Buoyancy is not present under weightlessness conditions, since the term $m g \cong 0$ and the vector of $F_{\mathrm{B}} \cong 0$.

For a hypothetical mammalian cell with a diameter of $10 \mu \mathrm{m}$ and a density $\rho$ of $1.05 \mathrm{~g} \mathrm{~cm}^{-3}$ in a liquid culture medium of $\rho=1.01$, the upwards directed buoyant force would be approximately:

$$
\begin{aligned}
F & =5.24 \times 10^{-10} \times 1.05 \times 9.81-\left(5.24 \times 10^{-10} 1.01\right) \\
& =4.87 \times 10^{-9} \mathrm{~N}
\end{aligned}
$$

Another phenomenon related to buoyancy but actually completely different is the Marangoni convection. It is the effect on mass transfer across a liquid-gas interface. The phenomenon of liquid flowing along an interface from places with low surface tension to places with a higher surface tension is named after the Italian physicist Carlo Giuseppe Matteo Marangoni (1840-1925). The Marangoni number is frequently expressed as:

$$
\mathrm{Ma} \cong\left[(-\delta \gamma / \delta c) c_{0} H\right] / \mu D
$$

In this equation, $(\delta \gamma / \delta c)$ is the dependence of surface tension on concentration, $c_{0}$ a characteristic concentration, $H$ a characteristic length and $D$ the diffusivity of the solute in the phase of interest. This Marangoni number expresses the ratio of a characteristic diffusion time and a characteristic time for Marangoni driven flow. Usually, the above mentioned buoyancy or Rayleigh effect dominates the flow in liquid layers with dimensions larger than $1 \mathrm{~cm}$. The Marangoni effect usually dominates when the characteristic dimension is smaller than $1 \mathrm{~mm}$. For chemical engineers, the layers of $1 \mathrm{~mm}$ are more relevant as these layers are more often encountered in mass transfer equipment. Under normal gravity, however, it is practically impossible to study the flow pattern in such thin layers - not even with the help of a sophisticated Laser-Doppler anemometer. Therefore, the only way to study Marangoni flows in $1 \mathrm{~cm}$ layers separately from buoyancy is by experiments in a reduced gravity environment. Although Marangoni convection does not 
change under microgravity conditions, the near weightlessness environment facilitates a better measurement of this phenomenon. For detailed information on the Marangoni effect see the academic thesis of T. Molenkamp, University of Groningen [22].

\subsection{7}

\section{Coriolis Acceleration}

Coriolis acceleration is a type of g-field that acts upon moving objects within a rotating system such as the Earth or a centrifuge. Coriolis accelerations are socalled cross-coupled responses, owing to the angular motion in two planes. It is an effect of rotation that contributes to the impurity of gravity generated by a rotating system. For gravitational research it is particularly involved in studies of moving objects, such as flagellates in hypergravity fields. In humans or animals, this acceleration is responsible for motion- and Space-sickness while moving in a rotating field, mediated by action on the inner ears' semicircular canals. The definition of this acceleration in $g \mathrm{~s}$ is:

$$
a_{\text {Coriolis }}=(2 v \times \omega) / g
$$

where $a_{\text {Coriolis }}=$ Coriolis acceleration expressed in $g, v=$ velocity of the moving object $\left(\mathrm{m} \mathrm{s}^{-1}\right), \omega=$ angular velocity of rotating system $\left(\mathrm{rad} \mathrm{s}^{-1}\right), \mathrm{g}=$ acceleration due to gravity $\left(\mathrm{m} \mathrm{s}^{-2}\right)$.

If the angular velocity is expressed in revolutions per minute (rpm), Eq. (22) changes to:

$$
a_{\text {Coriolis }}=(2 \pi v / 30 \times g) \times \mathrm{rpm}
$$

where $\mathrm{rpm}=$ revolutions per minute $=$ centrifuge speed.

The impact of the Coriolis force upon a sample depends on the axes along which the object moves in respect to the rotation axes. The highest impact is when the motion of the sample occurs in a plane of $90^{\circ}$ to the rotation axes.

The impact of this phenomenon is increased in a rapidly changing angular acceleration field such as centrifuges with relatively small diameters for generating a certain $g$-value compared with the velocity, $v$, of the object studied. The magnitude, rpm, of the primary acceleration is also important. Two rotating systems with different radii but spinning at similar rpms would generate the same Coriolis accelerations.

For example, for an object like the unicellular Loxodes moving at a speed of $200 \mathrm{um} \mathrm{s}^{-1}$ (R. Hemmersbach, DLR, Cologne, Germany, personal communication) in a rotating system of $100 \mathrm{rpm}$, the Coriolis acceleration would be $4.2 \times 10^{-4} \times \mathrm{g}$. If the rotation would generate a $g$-force of $1.0 \times \mathrm{g}$, the perturbation due to the Coriolis acceleration would be less than $0.05 \%$. Coriolis accelerations also play a role in rotating microgravity simulators such as the clinostat [23], the random positioning machine $[18,24]$ and the rotating wall vessel [25]. 


\section{References}

1 Minster, O., Innocenti, L., Mesland, D. Looking at Science on Board Eureca, pp. 3-6, ESA BR-80, ESA Publications Division, ESTEC, Noordwijk, 1993.

2 Pollard, E.C. J. Theor. Biol. 1965, 8, 113-123.

3 Albrecht-Buehler, G. ASGSB Bull. 1991, 4(2), 25-34.

4 Mattok, C. (Ed.), Biorack on Spacelab IML-1, ESA SP-1162, ESA Publications Division, ESTEC, Noordwijk, 1995.

5 Perry, M. (Ed.), Biorack on Spacehab: Experiments on Shuttle to Mir Missions 03, 05 Q 06 , ESA SP-1222, ESA Publications Division, ESTEC, Noordwijk, 1999.

6 Cogoli, A. (Ed.), Cell Biology and Biotechnology in Space. Advances Space Biology and Medicine, Elsevier, Amsterdam, 2002.

7 Burk, W.R. (Ed.), Biological Experiments on Bion-8 and Bion-9, ESA SP-1190, ESA Publications Division, ESTEC, Noordwijk, 1996.

8 Gaubert, F., Schmitt, D., Lapiere, C. Bouillon, R. (Eds.), Cell and molecular biology research in space, FASEB J. 1999, 13(Suppl.), S1-S178.

9 Häder, D-P., Hemmersbach, R., Lebert, M., Gravity and the Behavior of Unicellular Organisms. Chapter 4.2, Loxodes, pp. 67-74, Cambridge University Press, Cambridge, 2006.

10 Marthy, H-J. (Ed.). Developmental Biology Research in Space, Elsevier, Amsterdam, 2003.

11 Moore, D., Cogoli, A., Gravitational and space biology at the cellular level, in: Biological and Medical Research in Space: An Overview of Life Sciences Research in Microgravity, Moore, D., Bie, P., Oser, H. (Eds.), pp. 1-106, Springer, Berlin, 1996.

12 Longdon, N., David, V. (Eds.), Biorack on Spacelab D-1, ESA SP-1091, ESA Publications Division, ESTEC, Noordwijk, 1987.
13 van Loon, J.J.W.A., Folgering, E.H.T.E., Bouten, C.V.C., Veldhuijzen, J.P., Smit, T. H. ASME J. Biomech. Eng. 2003, 125(3), 342-346.

14 van Loon, J.J.W.A., Folgering, E.H.T.E., Bouten, C.V.C., Smit, T.H. J. Grav. Phys. 2004, 11(1), 29-38.

15 Patrick, C.W., Sampath, R., McIntire, L.V. Fluid shear stress effects on cellular function, in: Biomedical Research Handbook: Tissue Engineering, Palsson, B., Hubbell, J.A. (Eds.), pp. 1626-1645, CRC Press, Cleveland, Ohio, 1995

16 Klein Nulend, J., Van der Plas, A., Semeins, C.M., Ajubi, N.E., Frongos, J.A., Nijweide, P.J., Burger, E.H. FASEB J. 1995, 9, 441-445.

17 Weinbaum, S., Cowin, S.C., Zeng, Y. J. Biomech. 1994, 27, 339-360.

18 van Loon, J.J.W.A. Adv. Space Res. 2007, 39, 1161-1165.

19 Blancaflor, E.B., Masson, P.H. Plant Physiol. 2003, 133(4), 1677-1690.

20 Inoue, H., Nakamura, O., Duan, Y., Hiraki, Y., Sakuda, M.J. Dent. Res. 1993, 72(9), 1351-1355.

21 Ling, G. In Search of the Physical Basis of Life, p. 115, Plenum Press, New York, 1984.

22 Molenkamp, T. Marangoni Convection, Mass Transfer and Microgravity, Academic Thesis, Rijksuniversiteit Groningen, 6 November 1998.

23 Briegleb, W. ASGSB Bull. 1992, 5(2), 23-30.

24 Mesland, D.A.M. ESA Microgravity News 1996, 9(1), 5-10.

25 Hammond, T.G., Hammond, J.M. Am. J. Physiol. Renal Physiol. 2001, 281(1), F12-F25.

26 Weast, R.C. (Ed.), Handbook of Chemistry and Physics, 51 $1^{\text {st }}$ edition, The Chemical Rubber Co., Cleveland, Ohio, 1970-1971.

27 Todd, P. ASGSB Bull. 1989, 2, 95-113. 


\section{Further Reading}

Albrecht-Buehler, G., The simulation of microgravity conditions on the ground, ASGSB Bull. 1992, 5(2), 3-10.

Clément, G., Slenzka, K. (Eds.) Fundamentals of Space Biology: Research on Cells, Animals, and Plants in Space, Springer, New York, 2006.

DeHart, R.L., Davis, J.R. (Eds.), Fundamentals Step of Aerospace Medicine, pp. 346-347, Lea \& Febiger, Philadelphia, 1985.

Mihatov, L. (Ed.), General Physics, PrenticeHall Inc., Englewood Cliffs, New Jersey, 1984.
Marion, J.B. General Physics with Bioscience Assays, John Wiley \& Sons Inc., New York, 1979.

Sober, H.E. (Ed.), Handbook of Biochemistry Selected Data for Molecular Biology, $2^{\text {nd }}$ edition, The Chemical Rubber Co., Cleveland, Ohio, 1970.

Stephenson, R.J. Mechanics and Properties of Matter, $3^{\text {rd }}$ edition, pp. 14-17, John Wiley \& Sons Inc., New York, 1969. 